\title{
FRICTION-DRAG REDUCTION BY TRANSVERSE WALL MOTION - A REVIEW
}

\author{
Michael A. Leschziner \\ Imperial College London
}

\begin{abstract}
The quest for drag reduction is driven by environmental concerns, in general, and the need to reduce fuel consumption in transport applications, in particular. Turbulent friction is especially important in civil aviation, accounting for over $50 \%$ of the total drag in cruise. In this context, spatially and/or temporally varying in-plane wall motion, while undoubtedly difficult to implement in practice, has attracted major interest, because of the large drag-reduction margins it yields. It is also a forcing method that is of fundamental interest, as it provokes intriguing interactions between the spanwise Stokes layer induced by the wall motion and the near-wall turbulence-regeneration mechanisms. This article provides a relatively brief, 'entry-level', review of research in this area, principally over the past two decades. While far from being exhaustive, the review conveys a reasonably detailed picture of some major physical issues as well as of the outcome of the most important computational and experimental studies. Particular emphasis is placed on the question of how results obtained in idealised laboratory conditions and by simulation at relatively low Reynolds-number values pertain to high values typical of high-speed transport.
\end{abstract}

Keywords: Wall friction, turbulent drag, oscillatory wall motion, flow control.

\section{INTRODUCTION}

One of the most disadvantageous consequences of turbulence in any fluid flow over a solid surface is its propensity to substantially increase the skin friction and thus the energy required to either move the fluid - e.g. in a pipe - or the surface relative to the fluid - e.g. an aircraft wing. In simple terms, the main reason for this increase in friction is that turbulence close to the wall is in the form of chaotic quasi-streamwise vortices, formed from sweeps and ejections, which enhance momentum mixing near the wall and thus cause steep velocity gradients (i.e. intense shear straining) as the flow is forced to meet the no-slip condition at the wall. While this process may be desirable in situations in which flow separation is to be prevented - e.g. in near-stall conditions on a wing - it is distinctly undesirable in attached, low-curvature flow not subjected to a strongly adverse pressure gradient. Fig. 1(a), taken from a DNS by Touber and Leschziner (2012), is a snapshot of the transverse velocity fluctuations in a plane normal to the wall in a channel, and this shows clearly the vortical near-wall fluctuations within a layer of thickness $y^{+} \equiv \frac{y u_{\tau}}{v}<100$, where $u_{\tau} \equiv \sqrt{\tau_{w} / \rho}$ is the friction velocity, $\tau_{w}$ is the wall shear stress and $v$ and $\rho$ are the kinematic viscosity and density of the fluid, respectively. Fig. 1(b) reflects the effects of active control by oscillatory spanwise motion, and this will be discussed later. One consequence of the momentum transport caused by the motions seen in Fig. 1(a) is the formation of "streaks" - elongated regions of low- and high-streamwise-velocity fluctuations, illustrated in Fig. 1(c) by a snapshot of the streamwise velocity fluctuations across a wall-parallel plane at a wall-normal distance $y^{+} \simeq 6$, again taken from a DNS by Touber and Leschziner (2012). It is well established that the statistical average distance between either high-velocity or low-velocity streaks is of order $\Delta z^{+} \simeq 100$, while the length of the streaks is of order $\Delta x^{+} \simeq 1000$. In Fig. 1(c), the width and length of the domain are, respectively, $L_{z}^{+} \simeq 3000$ and $L_{x}^{+} \simeq 6000$.

It is readily appreciated that a reduction in frictional drag in a turbulent flow must rely, in principle, on a reduction in the turbulence activity as that illustrated by Fig. 1. There are, unfortunately, very few ways of doing so, however.

With methods applicable only to certain liquids being excluded - e.g. the addition of polymers to, or the use of super-hydrophobic surfaces in water - the only passive drag-reduction method proven to be effective in any fluid flow is to cover the wall bounding the flow with streamwise-oriented (or possibly herringbone-shaped) riblets (e.g. Bechert et al, 1997, Nugroho et al, 2013). In carefully controlled conditions and with carefully optimised shapes, riblets yield maximum drag-reduction

\footnotetext{
${ }^{*}$ Corresponding author (M. A. Leschziner, mike.leschziner@ic.ac.uk)
} 


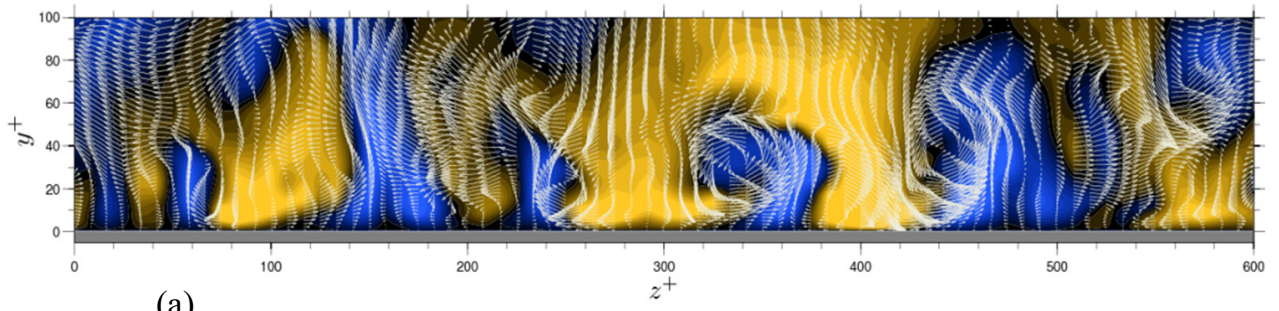

(a)

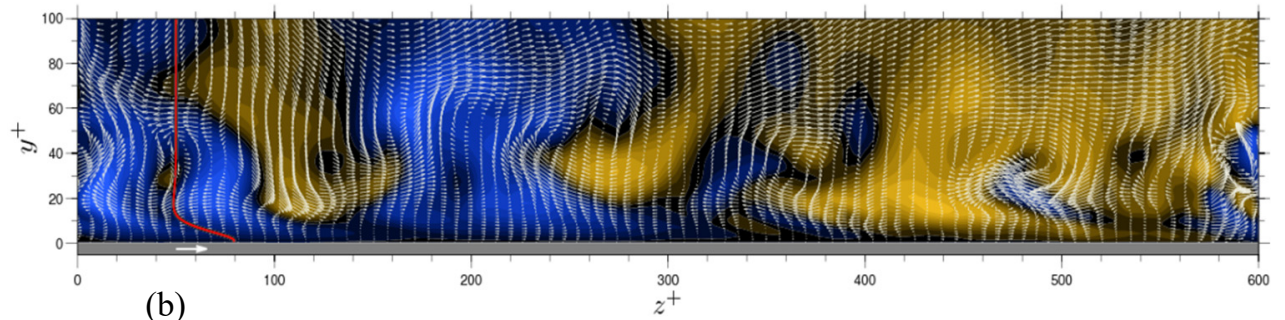

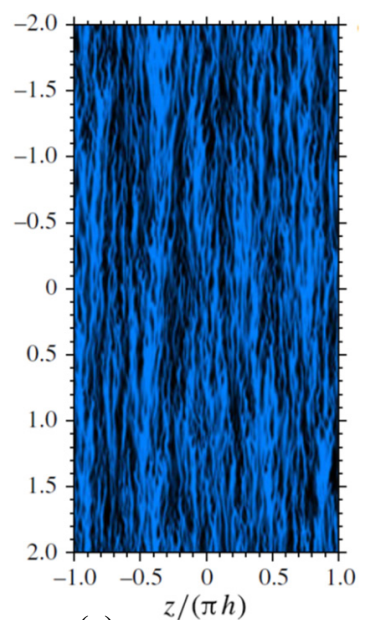

(c)

Fig. 1 Turbulence structure near a wall: (a) snapshot of transverse motions on a wall-normal plane (ejections, sweep and quasi-streamwise vortices) in canonical channel flow at $R e_{\tau}=\frac{u_{\tau} h}{v}=500$, where $h$ is the channel half height; (b) as (a) but with oscillatory spanwise wall actuation at $T^{+}=\frac{T u_{\tau}^{2}}{v} \simeq 100$; (c) streaky structure (streamwise velocity fluctuations) across wall-normal plane at $R e_{\tau}=500, y^{+} \simeq 6$. Taken from Touber \& Leschziner (2012), with permission from Cambridge University Press.

levels of order $7-10 \%$. The riblets need to be extremely small, however, with height and inter-riblet dimensions being, typically, $\Delta y^{+}, \Delta z^{+}=O(15)$, and their ridges have to be kept very sharp to avoid a steep deterioration in performance. These constraints, as well as manufacturing and maintenance difficulties, have prevented the uptake of riblets in practice.

Active control methods include blowing (high-rate transpiration), the imposition of cross-flow motion by Lorenz body forcing (in electrolytic liquids), use of plasma electrodes and the imposition of transverse and/or wavy wall motion. As blowing adds mass to the flow, the reduction in friction drag is achieve by displacing high-momentum fluid away from the wall, and this is accompanied by increased form drag, the latter negating the former. The other methods (except those involving the motion of wavy wall undulation) are all closely related, insofar as all are designed to provoke a transverse flow component close to wall, which disrupts the cycle that sustains near-wall turbulence.

There are currently very few convincing examples of plasma actuation yielding friction-drag reduction (Duong et al, 2013 being one), while the effectiveness of Lorenz forcing has only been demonstrated in isolated cases (e.g. Du et al. 2002; Pang \& Choi 2004). The large majority of numerical as well as experimental studies targeting friction-drag reduction (there are about 50 journal papers on this subject, going back to Jung et al, 1992) involved the imposition of oscillatory (usually) in-plane wall motion, either in channel flow or in spatially evolving boundary layers. A recent alternative proposal is the use of a dense array of rotating or oscillating discs embedded flush with the wall (Ricco \& Hahn, 2018), and this arrangement is shown to yield moderate drag-reduction margins, not drastically lower than oscillatory spanwise motion. The review contained in the following sections restricts itself to control by in-plane wall motion.

\section{DRAG REDUCTION - QUANTIFICATION AND MECHANISMS}

\subsection{Overview}

Early indications that spanwise sweeping near-wall flow can give rise to drag reduction were provided by experiments and simulations of 3D wall-bounded flows by Bradshaw \& Pontikos (1985) and Moin et al (1990), respectively. Such motions, if occurring in a manner that gives rise to significant spatial and/or temporal variations in the near-wall flow direction, disrupt the ability of the shear strain to sustain the mechanisms that are responsible for the turbulence-regeneration cycle, and they result in a weakening of the near-wall streaks. An especially illuminating demonstration of the above process is provided by a DNS of Howard \& Sandham (2000) in which a fully developed channel flow was suddenly subjected to a sustained (constant) spanwise wall motion. This sudden forcing was shown to result in a drop in turbulence activity and drag. However, the drop persisted only for a relatively short transient phase, after which the turbulence recovered to a canonical state compatible with the resultant shear following its re-orientation. Thus, the 


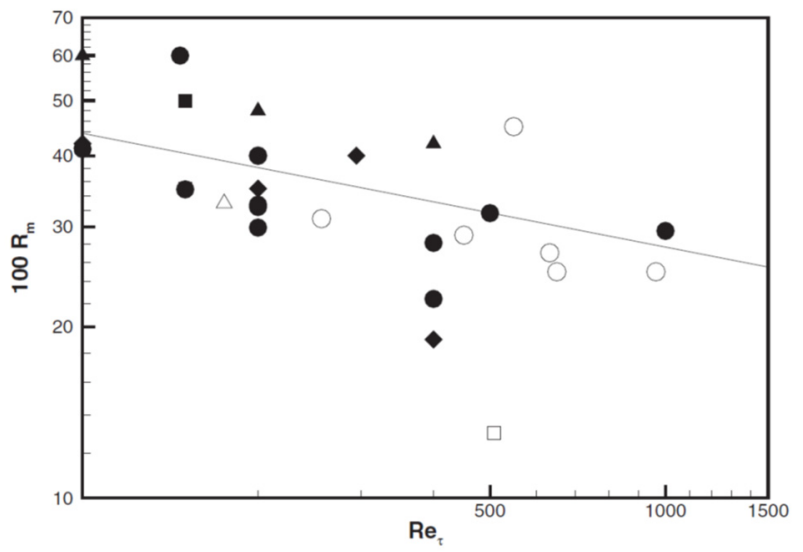

Fig. 2 Compilation of gross drag-reduction levels by wall actuation in a variety of geometries and for various combinations of actuation parameters (spanwise wall motion, streamwise- and spanwise travelling waves, Lorenz forcing). The straight line represents $R_{m} \propto R e_{\tau}^{-0.2}$. Taken from Gatti \& Quadrio (2013), with permission from AIP Publishing.

drag reduction was demonstrated to arise due to the unsteady directional change in near-wall straining.

Drag reduction by spanwise wall motion has been intensively investigated, mostly by means of channel-flow DNS, by Quadrio and his associates (Baron \& Quadrio, 1996, Quadrio \& Ricco, 2004, Quadrio et al, 2009, Viotti et al, 2009, Quadrio \& Ricco, 2011 and Gatti \& Quadrio, 2013, 2016 - among many other publications). Other notable studies are those of Touber \& Leschziner (2012), Agostini et al (2014) and Hurst et al (2014). Efforts based on DNS for spatially evolving boundary layer have been led by Skote (e.g. Skote, 2011, 2013 and Skote et al, 2015), and these will be the subject of Section 2.3. There have also been about 15 experimental studies on boundary layers and pipe flows (e.g. Laadhari et al, 1994, Choi \& Graham, 1998, Ricco \& Wu, 2004, Gatti et al, 2015, Bird et al, 2018a,b), reported to yield drag reductions in the range $16 \%-45 \%$.

Fig. 2, taken from Gatti \& Quadrio (2013), provides a compilation of gross drag-reduction margins reported in computational and experimental studies for a variety of wall-actuation scenarios and parameters, including spanwise and streamwise wall-travelling waves and Lorenz-force actuation. As is seen, most drag-reduction values cluster around $30-40 \%$, with outliers of $50 \%+$ being due to very high wall-velocity values or "pumping action' by travelling wavy-wall deformations. The straight line represents $R_{m} \propto R e_{\tau}^{-0.2} \quad$ (Reynold-number dependence will be discussed in Section 3).

While these are clearly very large reductions in drag, it has to be borne in mind that the benefits are significantly counteracted by the penalties of expending power to actuate the wall. This will be an issue considered in Section 2.2 below.

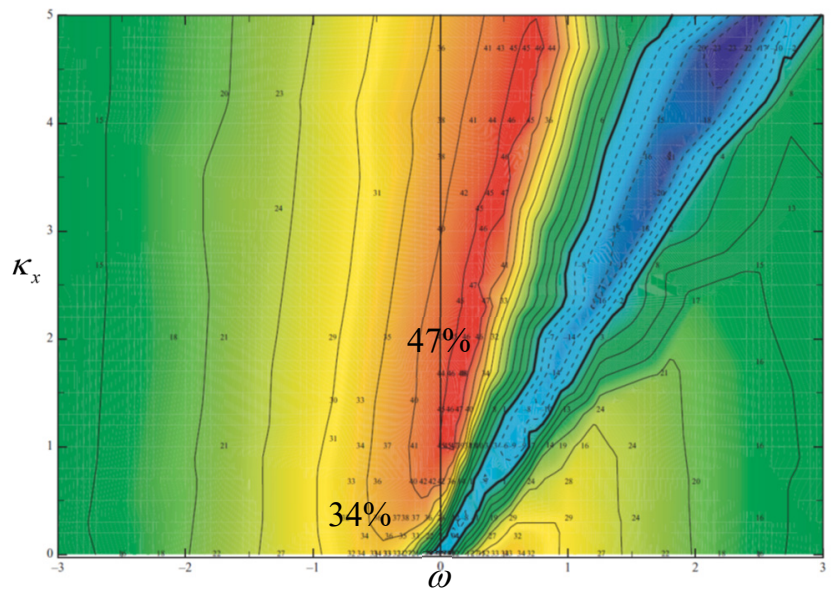

Fig. 3 Gross-drag-reduction map, in terms of oscillation frequency and streamwise wave number, for channel flow at $R e_{\tau}=200, \hat{W}^{+}=12$. The frequency and wave number are here scaled by the channel height and centreline velocity, rather than by the friction velocity. Red colour indicates drag reduction and blue drag increase. The maximum value is $47 \%$. For pure spanwise oscillations (abscissa), the maximum value is 34\%. Taken from Quadrio et al (2009), with permission from Cambridge University Press.

\subsection{Channel flow}

One outcome of hundreds of simulations performed by Quadrio's group (Quadrio et al, 2009) for channel flow over relatively small domains at $R e_{\tau}=200$ subjected to oscillatory wall motion is the (now, near-iconic) 'Quadrio Map' shown in Fig. 3. Here, the actuation is governed by $W^{+}=\hat{W}^{+} \cos \left(\frac{2 \pi t^{+}}{T^{+}}+\frac{2 \pi x^{+}}{\lambda_{x}^{+}}\right)$, where $\hat{W}^{+}(=12)$ is the wall-velocity amplitude, $\omega^{+}=\frac{2 \pi}{T^{+}}$is the oscillation frequency, and $\kappa_{x}^{+}=\frac{2 \pi}{\lambda_{x}^{+}}$is the wave number of the streamwise travelling wave. The superscript ' + ' indicates wall-scaling, based on the friction velocity $u_{\tau, o}$ of the unactuated boundary layer. While this map (in which the frequency and wave number are scaled by the channel height and centreline velocity, rather than by the friction velocity) is obviously not representative of all actuation scenarios - different $\hat{W}^{+}$, higher $R e_{\tau}$, boundary layers, in particular - it nevertheless gives a fair picture of the complex sensitivity of the drag to the actuation parameters - especially the proximity of the drag increase and the drag-reduction regions. The maximum drag reduction is seen to around $47 \%$. Two other interesting maxima are $45 \%$ for $\omega^{+}=0$, i.e. a standing-wave motion, and $37 \%$ for $\kappa_{x}^{+}=0$, i.e. streamwise-homogeneous ("pure") oscillatory motion. The latter maximum occurs for the 


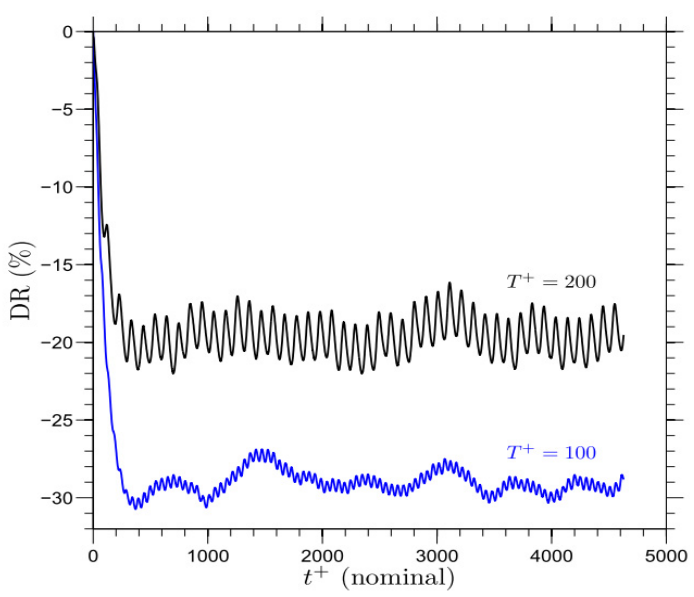

(a)

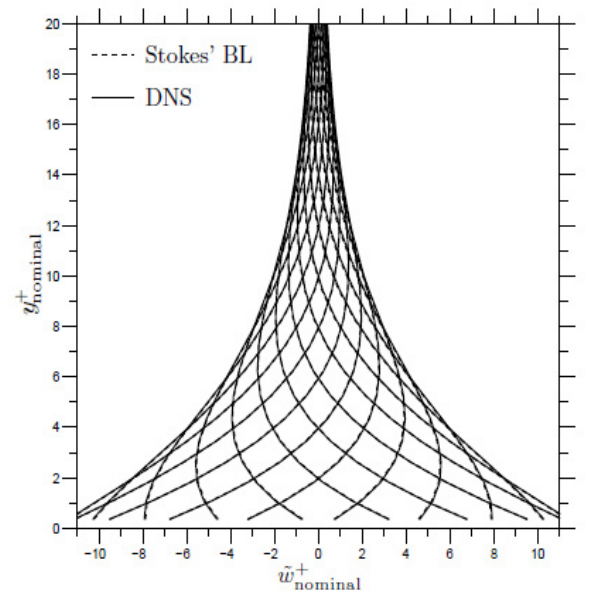

(b)

Fig. 4 Effects of spanwise wall oscillations on drag in channel flow: (a) wall-averaged drag reduction as a function of time for two actuation periods at $R e_{\tau}=1020$. Taken from Agostini et al (2014), with permission from Cambridge University Press; (b) profiles of spanwise velocity during the actuation cycle at $\operatorname{Re}_{\tau}=500$ and $T^{+} \simeq 100$. Taken from Touber \& Leschziner (2012), with permission from Cambridge University Press.

actuation period $T^{+} \approx 100$, which corresponds to values in the range $T^{+}=50-80$ when normalisation is effected with the actual, rather than nominal (unactuated), friction velocity. The value $T^{+} \simeq 100$ is often referred to as the 'optimal' or 'near-optimal' actuation period. Interestingly, this near-optimum appears to apply, at least approximately, over a wide range of conditions, including in boundary layers and for different $\hat{W}^{+}$and $R e_{\tau}$, the latter at least up to the maximum of 1600 and 2100 examined so far with DNS by Hurst et al (2014) and Gatti \& Quadrio (2013).

The broad applicability of the optimum $T^{+} \approx 100$ is curious and deserves discussion. A first set of observation may be made by reference to Fig. 4(a), taken from Agostini et al (2014). This shows the variation of the wall-averaged drag-reduction level for a channel flow at $R e_{\tau}=1020$ in which the wall is actuated at $\hat{W}^{+}=12$ and $T^{+}=100$ or 200 . At the lower value, the drag achieves its lowest level (30\%), and, importantly, the level is virtually independent of the actuation cycle. This is due to the fact that the Stokes layer - the layer containing the transverse motion whose velocity profiles at different phases is shown in Fig. 4(b) - is virtually confined to the viscous layer, $y^{+}<12$. The Stoke strain does not, therefore, contribute to the turbulence-production level in and above the buffer layer, and its effects are largely confined to a disruption of the regeneration cycle in the immediate proximity of the wall. Another explanation is provided by Blebois et al (2013) who use Generalised Optimum Perturbation theory to argue that the time scale for generating streaks in wall shear strain is $O(50)$, a value that fits well with the period scaled with the actual (true) wall shear stress. Animations derived from DNS data and reported in Touber \& Leschziner (2012) show that the cyclic change in strain due to the Stokes motion tends to severely weaken the streaks when the strain changes rapidly, while the streaks tend to re-establish themselves when the strain 'lingers' at times when the strain approaches its maximum value. This occurs twice during a cycle period, i.e. $\Delta t^{+}=T^{+} / 2=50$.

Efforts to explain the detailed and highly interactive physical mechanisms underlying the drag reduction are often complex and sometimes contradictory - or at least not mutually compatible. Alternative interpretations can be found in Touber \& Leschziner (2012), Blebois et al (2013), Ricco et al (2012), Yakeno et al (2014), Agostini et al (2014), Agostini et al (2015). The last paper, in particular, presents an elaborate and difficult to summarise paradigm that is based on the response of the turbulent vorticity components to the Stokes strain. An example of the contradictory nature of the interpretations is provided by the analysis of Agostini et al (2014) who examined phase-averaged statistics of the non-optimal actuation at $R e_{\tau}=1020$ and $T^{+}=200$. The dragreduction variations for this (intentionally chosen) nonoptimal case is shown in Fig. 4(a), and is seen to feature actuation-dependent, periodic, rise and fall of the dragreduction level. Agostini et al thus examined the behaviour of phase-averaged turbulence statistics, including the budgets of the Reynolds stresses, dissipation and enstrophy as the drag increases and decreases. This led them to conclude that the origin of the drag reduction and increase was a corresponding rise and fall in the turbulence production, while dissipation varied only modestly and in harmony with the variations in production. These observations, especially the harmony between the trends of drag and the dissipation, and the modest phasewise changes in the dissipation relative to other processes in the budget, are at odds with the mechanism proposed 


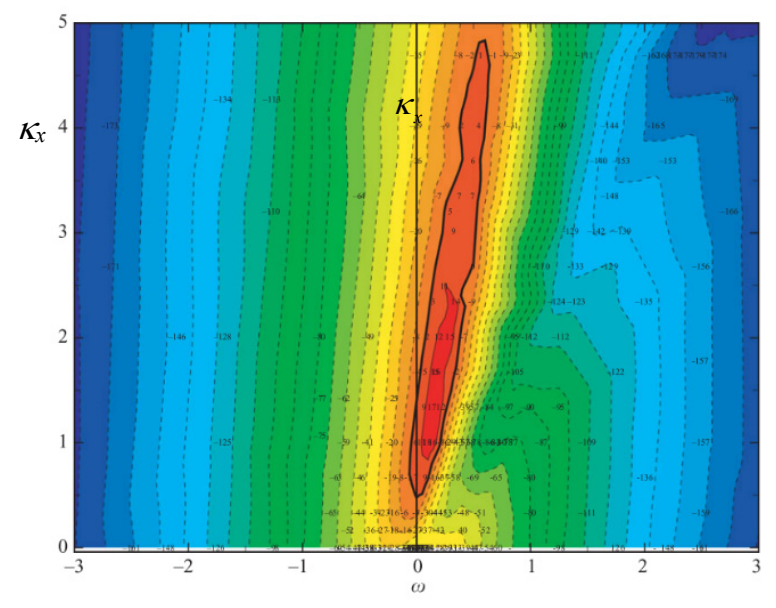

Fig. 5 Net-drag-reduction map, corresponding to Fig. 3. The maximum value is $17 \%$. For pure spanwise oscillations (abscissa), the maximum value is $-28 \%$. For the normalisation of the actuation parameters, see caption of Fig. 3. Taken from Quadrio et al (2009), with permission from Cambridge University Press.

by Ricco et al. (2012), which is based on the argument that the enstrophy is enhanced by the Stokes motion, and thus the increase in enstrophy and dissipation are held responsible for the drag reduction.

While the drag-reduction margins of $20-50 \%$ reported in the literature are very substantial, it must be borne in mind that these are gross levels, and do not take into account the power needed to impose the actuation. The question of what net benefits can be derived from active spanwise wall motion has rarely been investigated, partly because the engineering realisation of this type of active control appears too daunting. Almost uniquely, Quadrio et al (2009) have undertaken an extensive exercise of quantifying, subject to zero mechanical losses, the possible net power saving $P_{n e t \%}=P_{D R \%}-P_{\text {Actuation } \%}$. Fig. 5 shows the result corresponding to the map in Fig. 3.

As seen, the net saving is substantially negative, at $28 \%$, for pure spanwise oscillations (i.e. actuation quires more energy than that saved), but reaches $17 \%$ for a range of travelling-wave actuation (in Gatti \& Quadrio, 2013, the maximum is given as $14 \%$ ). In fact, this level peaks at around $30 \%$ when the wall-scaled amplitude of the spanwise wall velocity is reduced from $\hat{W}^{+}=12$ to 6 (Quadrio \& Viotti, 2016). For that lower value, pure oscillatory motion is also observed to yield a small positive net drag reduction, of order $5 \%$.

The question of whether the result in Fig. 5 suggests the possibility of ever realising some form of drag-reduction system using transverse wall motion in a practical environment remains entirely open. It certainly looks to be a daunting engineering challenge, even if mechanical losses can be kept insignificantly low, and questions are being asked whether plasma actuation with long streamwise electrodes or very thin spanwise synthetic

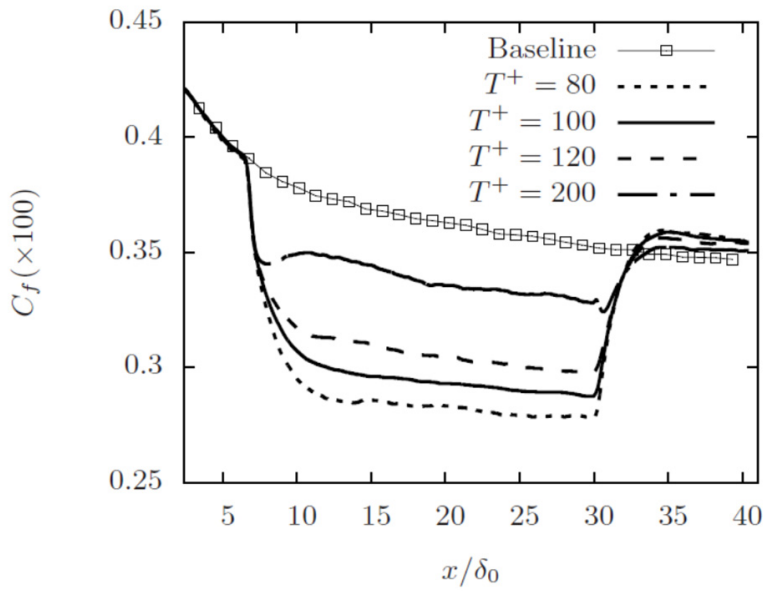

Fig. 6 Drag-reduction levels (in terms of friction factor) is a spatially-developing boundary layer subjected to pure spanwise wall oscillations over a restricted spanwise interval at different actuation periods at $R e_{\tau}=530$ at the start of the actuation. Taken from Lardeau \& Leschziner (2013), with permission from AIP Publishing.

surface-parallel jets can be configured to emulate the wall motion. One purely fluid-mechanical issue that needs to be answered first is whether the drag-reduction levels observed at low Reynolds numbers decline with increasing Reynolds number and, if so, at what rate. This question is addressed in the Section 3.

\subsection{Boundary layers}

Yet another issue to consider is whether the results observed in channel flow apply to spatially developing boundary layers. One problem with such boundary layers is that the skin friction varies significantly with the streamwise distance, so that a single set of actuation parameters will necessarily result in significant changes to their wall-scaled values. For example, a single oscillation period will result in a range of $T^{+}$values and therefore in non-optimal actuation conditions. DNS studies at any but low Reynolds numbers are rare (e.g. Skote et al, 2015, 2019). These show that, at least approximately, the drag-reduction levels observed in a channel also apply to boundary layers. An example is shown in Fig. 6. This is the result of boundary-layer simulations by Lardeau \& Leschziner (2013) at $R e_{\tau}=530$ the value at the position at which a purely oscillatory actuation is initiated. The actuation is effected in the streamwise segment $6.64 \delta_{0}-30.2 \delta_{0}$ where $\delta_{0}$ is the boundarylayer thickness at the start of the actuation. These simulations show a significant sensitivity to $T^{+}$, and a maximum drag reduction of about $28 \%$ at $T^{+}=80$. Also of interest is the observation that the drag decreases rapidly after the onset of the actuation, but recovers almost equally quickly within around 2 boundary-layer thicknesses. 

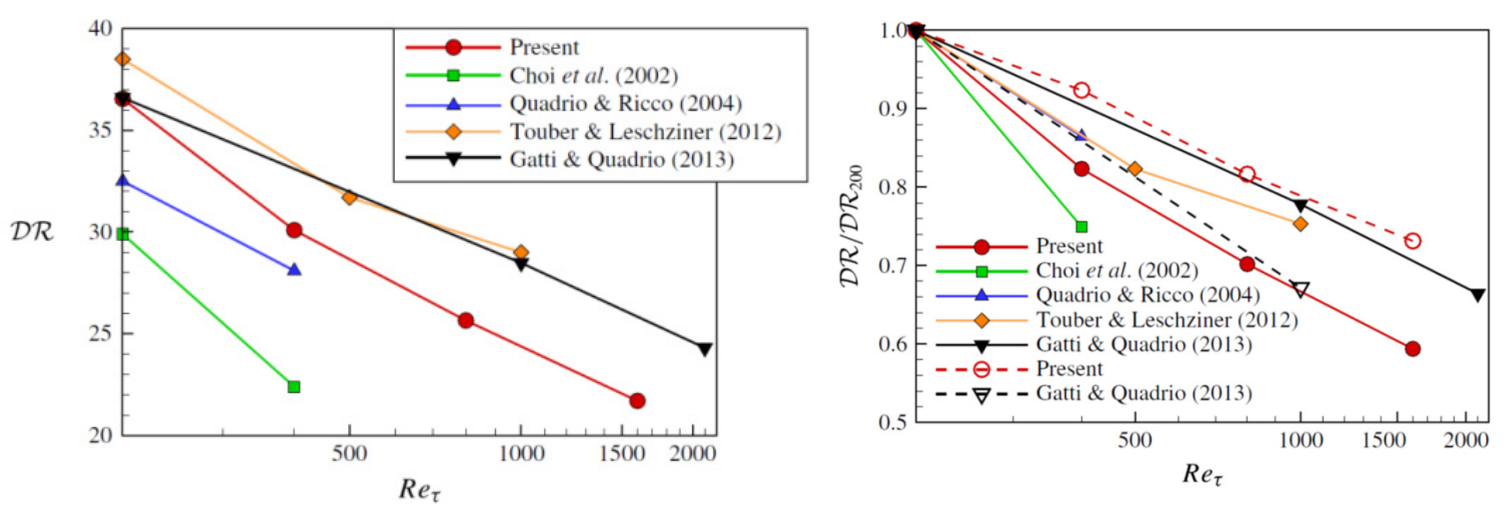

Fig. 7 Dependence of drag-reduction level on the Reynolds number: a compilation of results for various sets of actuation parameters. In the RHS plot, the values on the LHS plot are rescaled by the corresponding drag reduction level at $R e_{\tau}=200$. Taken from Hurst et al (2013), with permission from Cambridge University Press.

Experiments provide ample evidence that supports computational observations. In fact, because boundary layers are much easier to realise in a laboratory than a fully-developed channel flow, most of the experimental work on the effects of spanwise wall motion has been done on boundary layers (e.g. Choi, 2002, Ricco \& Wu, 2004, Bird et al, 2018a, b, among about a dozen others). A few experiments have also been performed on pipes and duct flows (e.g. Choi \& Graham, 1998, Auteri et al, 2010). While it is difficult to extract quantitatively meaningful comparisons from the experimental studies, because of the large variations in flow conditions (e.g. Reynolds number - usually stated in terms of momentum thickness) and actuation parameters, many of the experiments are reported as yielding yielded drag-reduction levels of the order 25-35\% (empty circles in Fig. 2), hence supporting, qualitatively, channel-flow and boundary-layer predictions.

\section{REYNOLDS-NUMBER DEPENDENCE}

\subsection{Global observations}

As noted in previous sections, there is unambiguous evidence from DNS studies for channel and boundarylayer flows that oscillatory spanwise wall motion yields gross drag-reduction levels approaching $50 \%$ and, potentially, net levels of order $20-30 \%$, depending upon the parameters of the actuation. However, the bulk of drag-reduction data derived from DNS are for low Reynolds-number values, which are not pertinent to realistic operational conditions. The reason is simple: the computational costs of DNS rise approximately in proportion to $R e_{\tau}^{3}$, given a constant domain size, because the length scale of the turbulent eddies that need to be resolved at the dissipative end of the eddy-size spectrum diminishes in proportion to $R e^{3 / 4}$, to which needs to be added the reduction in time scale.

In Section 2.2, Fig. 3 gives the gross drag-reduction map for $R e_{\tau}=200$. In contrast, typical values for large civilian aircraft operating at cruise condition are
$R e_{\tau}=O\left(10^{5}\right)$. The question of how the drag-reduction effectiveness - in terms of both the gross level and the net gain - varies with increasing Reynolds number is, therefore, of major interest from a practical perspective.

At the time of writing, the highest Reynolds-number value computed in channel flow with spanwise wall motion is $R e_{\tau}=2100$ (Gatti \& Quadrio, 2016), and there have been several other studies reporting simulations in the range $R e_{\tau}=1000-1600$ (Agostini et al, 2013, 2015, Hurst et al, 2016). However, these simulations whave been performed for isolated or narrowly defined types and ranges of actuation parameters and over substantially different box sizes. Such computations, when done over generously proportioned box sizes, require meshes of order 2 billion points to ensure sufficiently high fidelity. The costs are driven further upwards by the need to accommodate around 10 actuation periods and/or several travelling waves. The only way of reducing the extremely high costs is to restrict the box size, as done by Gatti \& Quadrio whose box size was $1.2 h \times 2 h \times 0.6 h$, but this is only possible for relatively short wave lengths, because the streamwise domain length must accommodate at least two waves. A substantial reduction in box size also leads to loss of fidelity through the exclusion of important large-scale motions in the outer portion of the boundary layer. As an aside, it is pertinent to note that the highest Reynolds numbers of canonical (unforced) channel flow computed are $R e_{\tau}=4000$ (Pirozzoli and Bernardini, 2013), $R e_{\tau}=4200$ (LozanoDuran \& Jimenez, 2014) $R e_{\tau}=5200$ (Lee et al, 2014) and $R e_{\tau}=8000$ (Yamamoto et al, 2018), while for canonical boundary layers, the maximum value reported is $R e_{\tau}=4000$ (Pirozzoli et al, 2013). To convey an inkling of the cost, it is remarked that the grid used for the highest value, $R e_{\tau}=8000$, contained 100 billion points, and grids of order 10-50 billion points have been adopted even at half of this value in efforts to achieve sufficiently high accuracy in respect of capturing the dissipative 


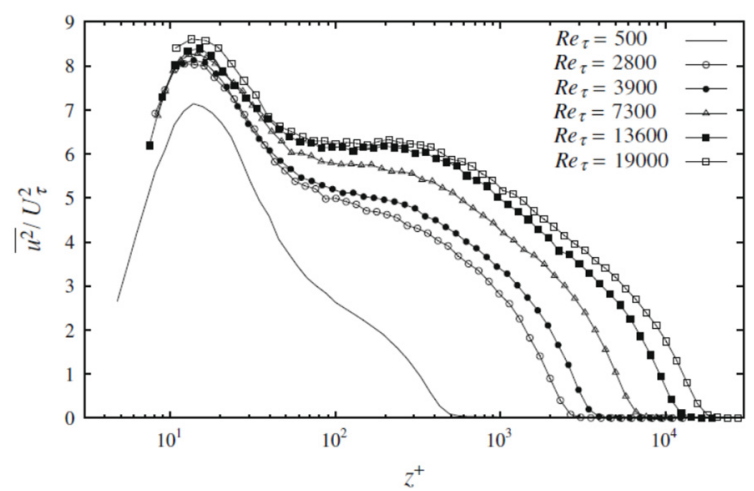

(a)

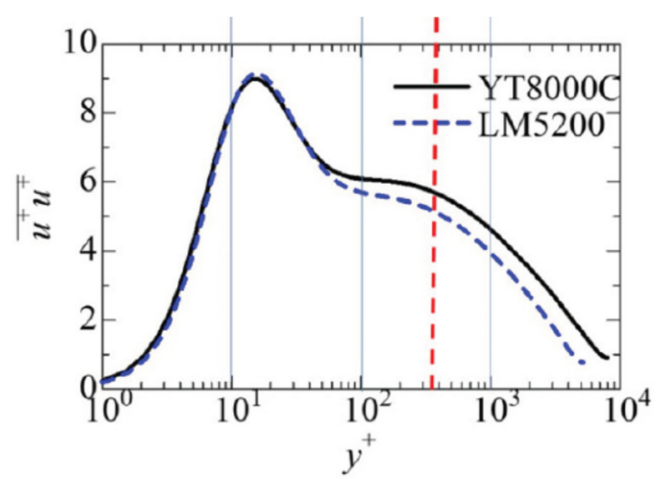

(b)

Fig. 8 Profiles of streamwise normal Reynolds stress at different Reynolds-number values: (a) experimental data for boundary layers, taken from Marusic et al (2010), with permission from Elsevier; (b) DNS data for $R e_{\tau}=4200,5300$ (Lee \& Moser, 2015, Yamamoto \& Tsuji, 2018).

small-scale motions at the Kolmogorov end of the eddysize spectrum.

While it is now well established that the drag-reduction effectiveness declines with Reynolds number, the variability of the combinations of actuation parameters adopted in simulations at Reynolds numbers higher than $R e_{\tau}=500$ has made it difficult to arrive at clear-cut conclusions about the rate of decline. Fig. 7, taken from Hurst et al (2013), provides a compilation of five sets of data. Each set arises from a different combination of actuation parameters, but all show a decline in dragreduction effectiveness.

Until around 2017, the general view was that the decline with Reynolds number followed roughly $D R \propto R e_{\tau}^{-(0.2-0.3)} \quad$ (see also Fig. 1). This trend was derived mainly from observations such as those in Fig. 7. However, more recently, careful DNS investigations with near-optimum travelling-wave actuation, implemented in relatively small channel-flow domains Gatti \& Quadrio (2016), have led to the conclusion that the decline is at a more modest rate, following a logarithmic path, as illustrated by the black line in Fig. 7. The origin and significance of this decline will be discussed in Section 3.3. Suffice it to say here that the logarithmic decline, if boldly extrapolated to flight Reynolds numbers, implies the potential of achieving net drag-reduction levels of order 10\% (Gatti \& Quadrio, 2016), assuming unrealistically, of course - that mechanical losses in activating the wall motion are negligible.

The question of the origin of the Reynolds-number dependence shown in Fig. 7 is undisputed, at least in broad principle: the turbulent scales above the viscous sublayer become more intense, their range grows and their impact on the viscous sublayer intensifies, making the sublayer thinner and less influential in terms of its contribution to the stress balance that dictates the skin friction. As a consequence, its modification by the control also becomes less influential. As the drag reduction is defined as $D R=\Delta C f / C f_{0}$, i.e. the ratio of skin-friction reduction and the skin friction of the uncontrolled boundary layer, and as
$C f_{0}$ declines with the Reynolds number, it follows that $\Delta C f$, which is induced by the actuation, falls faster than $C f_{0}$, and this implies a decline in the effectiveness with which the sublayer is modified by the wall motion.

The turbulence mechanisms which drive the dependence of the drag and the drag-reduction effectiveness on the Reynolds number are a matter of ongoing debate and are the subject of much current research, mostly on canonical, uncontrolled, channel flows and boundary layers. One predominant line of thought is that the autonomous formation of a particular set of energetic structures in the log layer plays an important role. A number of experiments by Marusic et al (2010), Mathis et al (2011) at the University of Melbourne and by Vallikivi et al (2015) in the Princeton University "Superpipe" have shown that the streamwise turbulence-energy component, scaled with the wall shear stress, rises substantially with Reynolds number towards a plateau or even, as some claim, a second maximum. This is illustrated in Fig. 8(a) for canonical boundary layers without actuation ( $z$ denotes, unconventionally, the wall-normal coordinate, normally denoted by $y$ ). The wall-normal position of the plateau is not well defined, but Marusic has proposed the correlation $y^{+}=3.9 \sqrt{R e_{\tau}}$ (Marusic et al, 2010), which is thus in the range $y^{+} \approx 200-400$. This rise in energy reflects the presence of large, streak-like, energetic, structures the formation of which is akin to that responsible for the formation of the small-scale streaks in the viscous layer - namely, ejections and sweeps forming quasi-streamwise vortices. DNS confirms the presence of the outer plateau, as shown by the profiles in Fig. 8(b) arising from channel-flow simulations at $R e_{\tau}=5200$ and 8000 , by Lee \& Moser (2015) and Yamamoto \&Tsuji (2018), respectively. The above super-streak interpretation of the outer structures is supported by the observation (Agostini \& Leschziner, 2019) that the statistical state of the turbulence in the layer populated by the structures displays an increasing trend, with rising Reynolds number, towards “one-component 

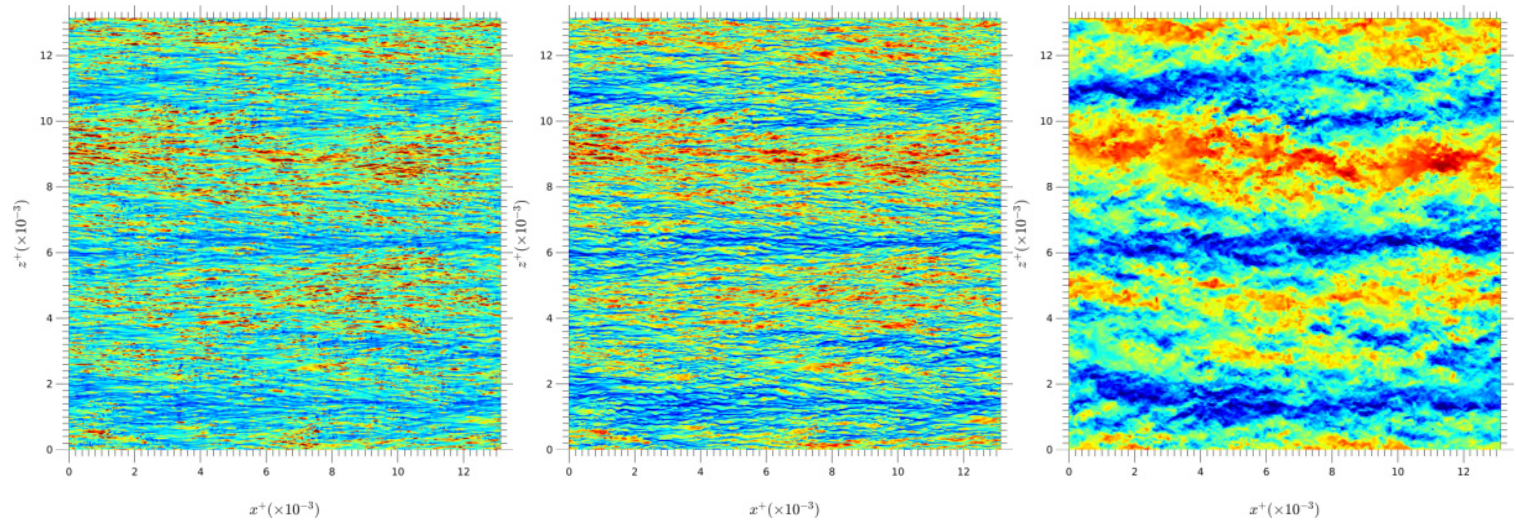

Fig. 9 DNS-derived snapshots of streamwise-velocity fluctuations on three wall parallel planes, $y^{+}=2,12$ and 200 (from left to right) for channel flow at $R e_{\tau} \simeq 4200$ at $y^{+} \simeq 12.5$ (see related study by Agostini et al, 2016).

turbulence"' - i.e. a state in which the Reynolds-stress tensor is increasingly dominated by one eigenvalue (or principal stress).

The question of why these outer structures should play a role in a control method whose effects are confined to the viscous sublayer requires a detailed examination of the near-wall turbulence structure, and this will be touched upon in Section 3.3. Here, it is merely noted that these structures are observed to affect the near-wall turbulence by way of three mechanisms: "footprinting", "modulation"” and "splatting", the first two introduced by Marusic et al (2010) and Mathis et al (2011), while the third was proposed and demonstrated by Agostini \& Leschziner (2014) and Agostini et al (2016). Footprinting is a process by which the large-scale motions of the outer structures induce corresponding convective motions near the wall, subject to a lag of order $\Delta x^{+} \approx 0.5 R e_{\tau}$. This is illustrated by Fig. 9, which shows three instantaneous fields of streamwise velocity fluctuations at $y^{+}=200$, 12 and 2, respectively, over a portion of the $x-z$ box of a channel-flow DNS by Lozano-Duran \& Jimenez (2014) at $R e_{\tau}=4200$. There is a visually obvious correlation between the field at $y^{+}=200$ and the near-wall fields. Modulation is a process by which the large-scale motions amplify or attenuate the small-scale turbulence near the wall, depending upon whether the large-scale fluctuations are positive or negative. Splatting is the most subtle mechanism of the three, and this causes the amplification and attenuation of the near-wall turbulence to be unequal. Positive footprints are accompanied by large-scale sweeping motions, which cause impingement (splatting) on the wall, while negative footprints are associated with large-scale ejections. Differences between the two cause asymmetric small-scale modulation, with amplification being stronger than attenuation.

In the context of drag reduction by wall motion, the above processes are expected to be especially influential. The key point here is that the control leads to a significant reduction in turbulence and in the strength of the near-wall streaks. As explained earlier, modulation entails an attenuation of the near-wall turbulence by the action of negative footprints. However, the streaks are already substantially weakened by the actuation, if this is efficiently implemented, so that the attenuation is marginal. On the other hand, the amplification by the positive footprints tends to be strong. Hence, the asymmetric modulation noted above in relation to splatting can be expected to be considerably more pronounced. Unfortunately, studies of the influence of outer structures on the near-wall physics in the presence of spanwise wall oscillations are rare. The most detailed is that of Agostini \& Leschziner (2018) for the case of streamwise homogeneous oscillatory spanwise motion at $R e_{\tau} \simeq 1000$ and $T^{+}=100$ and 200. This study will be discussed, among others, in Section 3.3

\subsection{Implications of log-law behaviour}

An observation made in many studies in which drag is reduced by some wall-based control, passive or active, is that the $\log$ portion of the profile $U^{+}=f\left(y^{+}\right)$, when normalised by the actual wall-shear velocity, is lifted, relative to the canonical case. This is illustrated in Fig. 10 (a) by way of two profiles for channel flow at $R e_{\tau}=1020$ and spanwise wall motion at two frequencies (Agostini et al, 2014). The plot provides an indication that the slope of the log portion may not be constant, and this point is also made in a DNS study by Skote (2014) of boundary layers, albeit at rather low Reynolds numbers at which the log law is very short and the slope (i.e. the inverse of the von Karman constant) is not self-evidently constant across $y^{+}$. There is little doubt that the main feature is the upward shift in the log law. Hurst et al (2016) report two sets of DNS-derived log-law distributions for two sets actuation conditions, one shown in Fig. 10(b), that suggest that the upward shift in the log law tends to become constant as the Reynolds number increases - i.e. independent of $R e_{\tau}$, given that all other actuation parameters remain invariant. Even if the not unimportant issue of the variability in the log-law slope is set aside, this statement needs to be viewed with caution, 


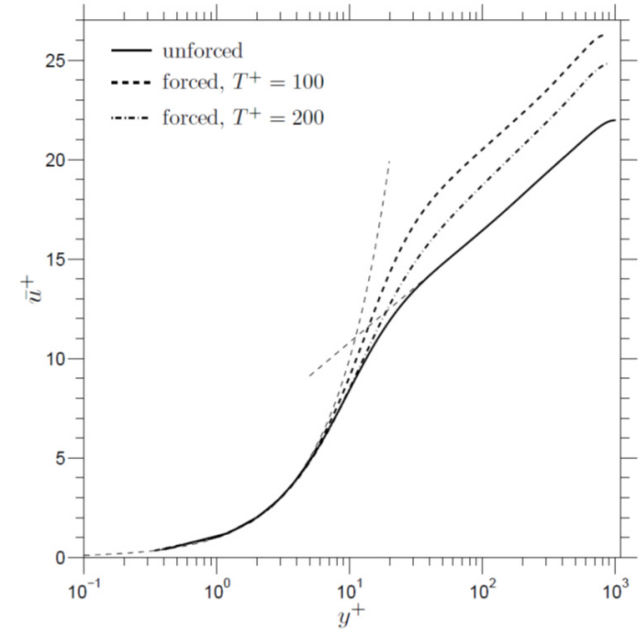

(a)

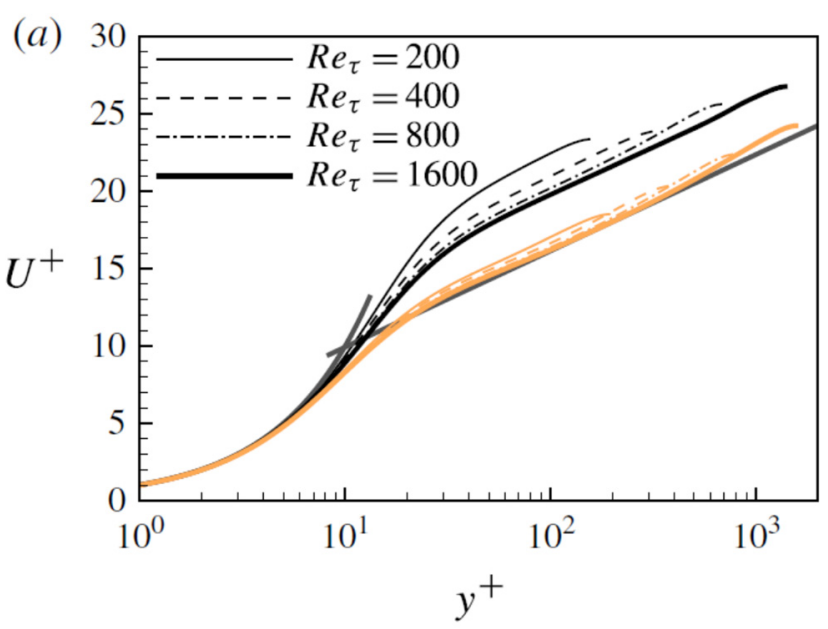

(b)

Fig. 10 Elevation of logarithmic velocity profile induced by oscillatory wall motion: (a) response to actuation period, channel flow at $R e_{\tau}=1020$, taken from Agostini et al (2014); (b) response to Reynolds number, channel flow at $T^{+} \simeq 100$, taken from Hurst et al (2014), with permission from Cambridge University Press.

because it is based, essentially, on two $R e_{\tau}$ values, 800 and 1600. Further related evidence is provided by Gatti \& Quadrio (2016), who report a large number of results for $R e_{\tau}=200-1000$ They argue that the von Karman constant is close to 0.39 for all Reynolds numbers, but here again, it must be pointed out that the range in $y^{+}$ over which the slope is approximately constant is very limited at low Reynolds numbers.

Simulations for spatially developing, actuated boundary layers by Skote (2014) and Skote et al (2015) similarly resulted in a distinctive upward shift in the log law, but the shift was observed to vary in the streamwise direction, thus suggesting that the shift is not constant as $R e_{\tau}$ changes. However, this investigation needs to be regarded with caution due to the fact that the maximum friction Reynolds number reached at the end of the simulation domain was below 700 .

If the shift is indeed assumed to be constant, it is possible to relate the drag reduction level to the Reynolds number and thus to extrapolate the reduction to an operationally relevant regime - a connection first proposed in Luchini (1996). To do so, we denote the shift by $\Delta U$, the velocity at the centre of the channel by $U_{h}$ and the velocity of the baseline (unactuated) flow by $U_{h, 0}$. The drag reduction, $D R$, can now be expressed as follows:

$$
\begin{aligned}
& D R=-\Delta C f / C f_{0} \\
& U_{h}=U_{h, 0}+\Delta U \\
& C f=\left(u_{\tau} / U_{h}\right)^{2}=2 / U_{h}^{+2} \\
& D R=1-\left(\frac{1}{1+\Delta U^{+} / U_{h, 0}^{+}}\right)^{2}
\end{aligned}
$$

Hence, if $\Delta U^{+}$is constant, $D R$ depends on $R e_{\tau}$ only via its link to $U_{h, 0}^{+}=\sqrt{2 / C f_{0}}$, which is $R e_{h, 0} / R e_{\tau, 0}$, the ratio of the Reynolds number based on the maximum velocity to the friction Reynolds number. To a firstorder accuracy, $R e_{h}$ is linearly related to $R e_{\tau}$ (in reality, the increase in $R e_{\tau}$ is slower than that of $R e_{h}$, as expressed by the Dean and Laadhari correlations (Dean, 1978, Laadhari, 2007). This then gives a nearly linear decline of $D R$ in a semi-log plot of $D R=f\left(R e_{\tau}\right)$. Thus, If $D R=30 \%$ at $R e_{\tau}=200$, for example, $D R$ reduced to approximately $20 \%$ at $R e_{\tau}=1000$.

Gatti \& Quadrio (2016) also show, subject to the assumption of constancy of log-law slope, that the friction factors (normalised with the mass-mean velocity) for the actuated and unactuated channel-flow cases are related by:

$$
\sqrt{\frac{2}{C f}}-\sqrt{\frac{2}{C f_{0}}}=\frac{1}{\kappa} \frac{R e_{\tau}}{R e_{\tau, 0}}+\Delta U^{+}
$$

Hence, if $\Delta U^{+}$is a constant offset, the actual friction factor (i.e the drag of the actuated flow) depends only on the friction factor of the baseline flow and the ratio of the friction-Reynolds-number values. Fig. 11, taken from Gatti \& Quadrio (2016) show the implied variation of the drag reduction levels $\mathfrak{R}=1-C f / C f_{0}$ starting from $R e_{\tau}=1000$, the various levels at that value arising from different actuation scenarios in terms of frequency, wave number and wall-velocity amplitude. The majority of studies on drag reduction have adopted a wall-velocity amplitude $\hat{W}^{+}=12$. For this value, Gatti \& Quadrio (2016) report a maximum gross drag reduction for the best travelling-wave scenario of $38 \%$ at $R e_{\tau}=1000$, and they 


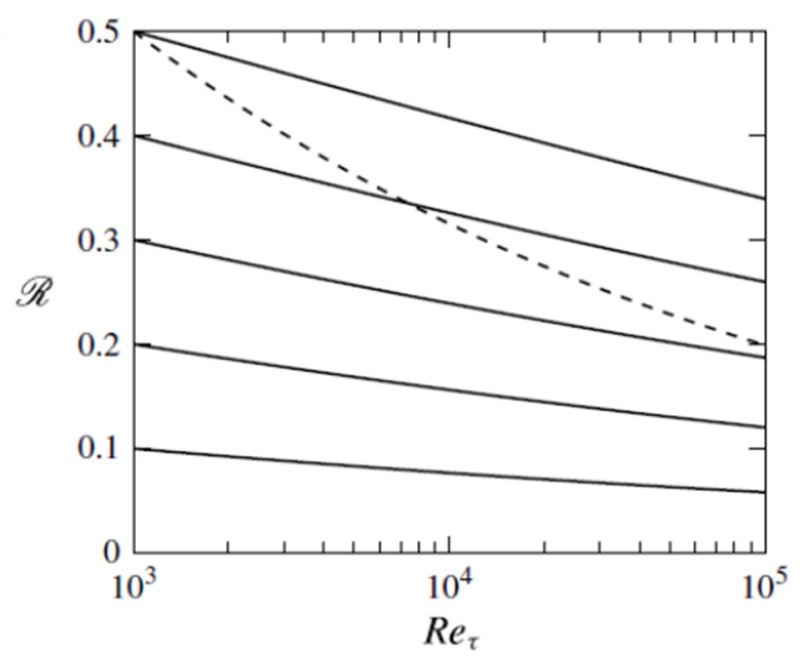

Fig. 11 Dependence of drag-reduction levels $\left(\mathfrak{R}=1-C f / C f_{0}\right)$ on friction Reynolds number. The dashed line represents a variation proportional to $R e_{\tau}^{-0.2}$; the solid lines are 'predictions' that arise from eq. (2) and assumed log-law shifts $\Delta U^{+}$at $R e_{\tau}=1000$. Taken from Gatti \& Quadrio (2016) with permission from Cambridge University Press.

show that the net drag reduction at this level is $19 \%$. Extrapolated to $R e_{\tau}=10^{5}$, this level reduces to around $10 \%$.

For a boundary layer, the situation is slightly more involved. Thus, the equivalent to eq. (2) can be shown to be

$$
\sqrt{\frac{2}{C f}}-\sqrt{\frac{2}{C f_{0}}}=\frac{1}{\kappa} \frac{R e_{\theta}}{R e_{\theta, 0}}+\Delta C
$$

where $R e_{\theta}$ is the momentum-thickness Reynolds number, and $\Delta C$ is a shift that depends on the shift of the log law and the shift in the wake region, which are not generally identical. The implication of eq. (3) is, therefore, that there is no clear-cut relationship between the Reynolds-number ratio and the drag reduction $D R$.

\subsection{The role of Re-dependent outer structures}

As observed in Section 3.1, a distinctive response to an increase in the Reynolds number is a progressive rise in the streamwise turbulence energy in the log layer, exemplified by Fig. 8. It is important to emphasise that this rise occurs in profiles that are scaled with the wall shear stress - i.e. scaling that presumes a universal behaviour of the near-wall turbulence. While the nearwall maximum, around $y^{+} \simeq 15$ is not quite universal either, the dependence of the energy in the outer layer is much more pronounced and indicative of strong departures from universality in terms of inner scaling. It is also this strong rise in energy that appears to offer a promising route to explaining the Reynolds-number- dependent decline in the drag-reduction effectiveness.

Unfortunately, a very small part of this literature deals the effects of outer structures on actuated flows, most studies analysing canonical channels flows and boundary layers. However, these studies are nevertheless pertinent to controlled flows, because they illuminated interactions that also must also apply to actuated flows. An argument supporting this assertion is that the wall motion only (or mostly) affects the turbulence within the viscosityaffected sublayer, but not the outer flow - except perhaps through global effects that arise from the reduction in $R e_{\tau}$ provoked by the actuation for a constant level of $R e_{\text {bulk }}$.

Any attempt to unravel the details of the interactions between the outer structures and the near-wall region - and hence the effect on the drag - requires access to detailed measurements or computational data extracted from DNS. Detailed experimental data targeting the role of outer structures have only been obtained for unactuated boundary layers with the aid of hot-wire probes (e.g. Marusic et al, 2010, Mathis et al, 2011, Baars et al, 2018). The data so extracted are in the form of time-series of streamwise turbulent signals at different wall-normal distances. In contrast, DNS data are in the form of fullvolume snapshots at relatively few time levels covering a relatively short period of time. In both cases, the data has to be decomposed into large-scale and small-scale components, to study the interactions between these scales, and there are a number of ways of doing so.

The choice of the small-scale/large-scale filter is not unambiguous, but not arbitrary either. This is exemplified by Fig. 12(a), which shows the streamwiseenergy density as a function of spanwise eddy size (wave length) and wall-normal distance for a channel flow at $R e_{\tau}=4200$ (Lozano-Duran \& Jimenez, 2014, Agostini

\& Leschziner, 2016). This spectrum provides clear evidence of the existence of two distinct regions of elevated energy - one, close to the wall, at around $y^{+} \simeq 15$, associated with the near-wall streaks, and the other is the outer region, at around $y^{+} \simeq 400$ indicative of the energetic outer structures. In terms of spanwise length scale, the inner peak, at around $\lambda_{z}^{+} \simeq 100$, is characteristic of the inter-streak distance in the buffer layer, while the outer peak is $\lambda_{z}^{+} \simeq 4000$, identifying "super-streaks" that are roughly one channel height apart. This spectrum thus suggests that a filter at around $\lambda_{z}^{+} \simeq 1000$ would be a reasonable choice. More elaborate approaches to scale decomposition are those of Zhang \& Chernyshenko (2016), based on a Pareto-front optimisation of the Fourier filter, of Baars et al (2016), based on a coherence function, in Fourier space, between the velocity signal at the outer location at which the largescale structures are observed and the velocity signal at any other wall-normal location, and of Agostini \& Leschziner (2014), in which a modal-decomposition formalism called "Empirical Mode Decomposition" has been used. The 


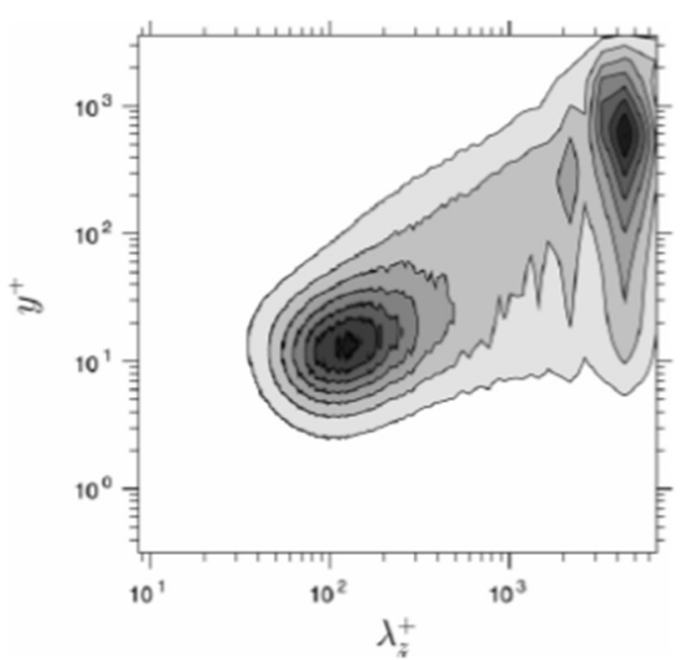

(a)

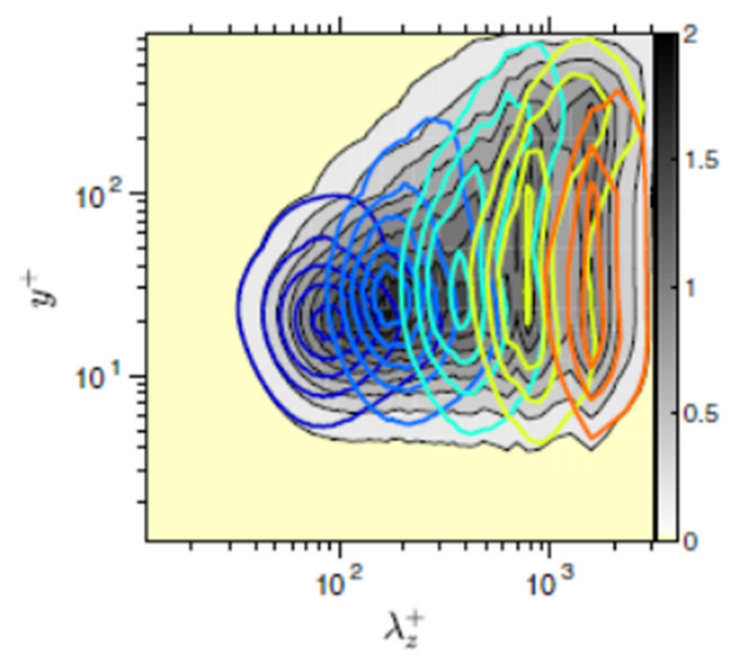

(b)

Fig. 12 Spectral maps of streamwise-energy fluctuations in spanwise direction: (a) canonical channel flow at $R e_{\tau}=4200$ by Lozano-Duran and Jimenez (2014), taken from Agostini \& Leschziner (2016) with permission from Cambridge University Press; (b) channel flow at $R e_{\tau}=1020$ actuated at $T^{+} \simeq 100$ with sub-spectra derived from the Empirical Mode Decomposition, taken from Agostini \& Leschziner (2018) with permission from Springer.

last approach decomposes any spatial two-dimensional field, derived from full-volume DNS realisations, into a series of narrow-wave-length-spectrum fields or "intrinsic modes", as illustrated by Fig. 12(b) which arises from channel flow at $R e_{\tau}=1020$ subjected to oscillatory wall motion at $T^{+}=100$ (this is discussed below in more detail). Each mode represents a relatively narrow range of length scales. These are then collected into two groups of small-scale and large-scale contributions, a process that require judgement much like the choice of a single Fourier filter to separate small-scale from large-scale components in a temporal signal. This is the only method that has been applied, so far, to the analysis of actuated flows (Agostini \& Leschziner, 2018), as discussed below.

Once the flow has been decomposed into small-scale and large-scale fields, it is possible to investigate how largescale fluctuations in the log layer are correlated with largescale fluctuations close to wall - the latter being "footprints" of the former - and how the intensity of the small-scale fluctuations near the wall responds to the outer large-scale fluctuations - referred to as "modulation". Reference to Fig. 12 shows that the energy of the large-scale structures extends right down into the viscous layer, and two-point correlations (Agostini \& Leschziner, 2014) confirm that large-scale fluctuations at the wall (the footprints) are closely correlated with the outer large-scale fluctuations. To establish the correlation between the large scales and the intensity of the small scales (the modulation), the envelope of the small-scale signal components needs to be determined. This is conventionally done by use of a method proposed by Mathis (Mathis et al, 2011) in which the Hilbert Transform is used in combination with lowpass filtering (Agostini et al, 2016). A key study that quantifies the role of footprinting and modulation in canonical flows is that of Mathis et al (2011).

The only study in which footprinting and modulation were analysed in actuated flows is that of Agostini and Leschziner (2018). The study examines DNS data for channel flow at the nominal (unactuated) Reynolds-number value $R e_{\tau}=1020$ at two actuation periods, $T^{+}=100$ and 200 without any streamwise waves. Although this is, energetically, an entirely unpromising case - as the actuation power is demonstrably higher that the power saved by the gross drag reduction of $30 \%$ and $20 \%$ at the two actuation periods, respectively - this actuation mode is tolerably simple in terms of the statistical processing that is needed to derive information on the direct and indirect contributions of footprinting and modulation of the outer structures on the drag.

As noted already, Agostini \& Leschziner used the Empirical Mode Decomposition (EMD) to separate the large-scale from the small-scale fields. Fig. 12(b) shows a decomposition into $6(5+$ residual $)$ modes by way of the streamwise-energy spectra in the spanwise-wavelength/wallnormal plane. Each mode covers a fairly narrow range of scales. Modes 1 and 2 - the left-most modes in Fig. 12(b) - are deemed to represent the small scales, while modes 46 are regarded as representing the large scales. Here again, it is important to note that the energy of the large scales extend right down to the viscous sublayer. Mode 3 represents intermediate scales and is excluded from the attribution. Fig. 10(b) also highlights one important limitation of the study - namely, the absence of a distinct scale-separation region, which is clearly present in Fig. 12(a) for the much higher Reynolds number. In fact, it is arguable that $R e_{\tau} \simeq 1000$ is the lowest Reynolds number at which the interactions at issue can be studied, and it needs to be noted that the actual Reynolds number is even 


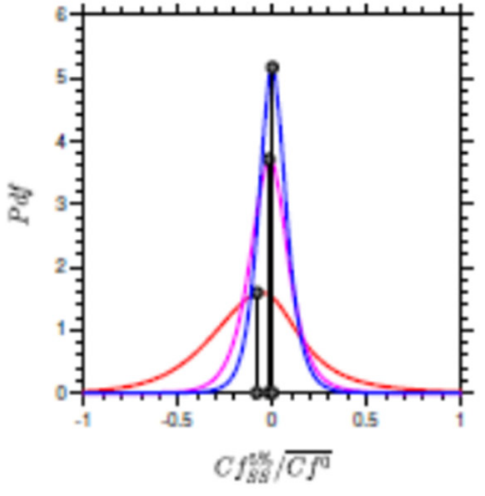

(a)

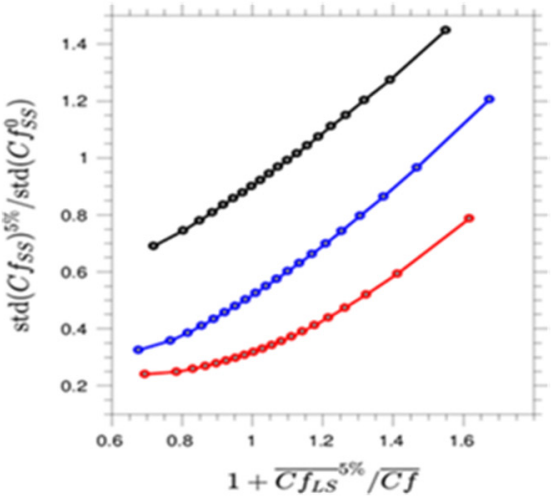

(b)

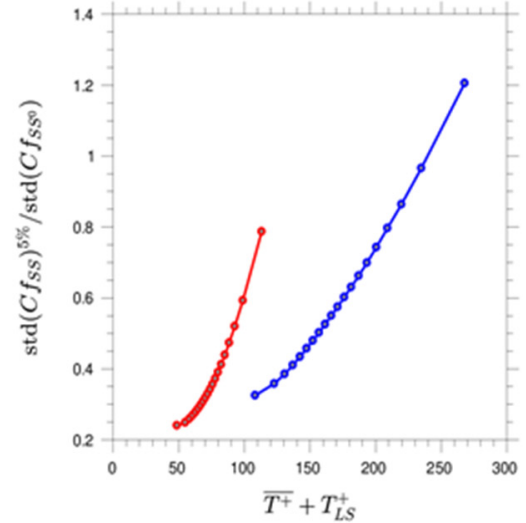

(c)

Fig. 13 Effects of large-scale skin-friction footprints on the intensity of the small-scale skin-friction fluctuations for channel flow at $R e_{\tau}=1020$ : (a) PDFs of fluctuations for the $5 \%$ strongest positive footprints (red), $5 \%$ strongest negative footprints (blue), negligible footprints (magenta) at $T^{+} \simeq 100$; (b) standard deviations of the smallscale friction fluctuations as a function of the intensity of the large-scale footprints ( $5 \%$ intervals of strength) for the nominal periods $T^{+}=100$ (red), 200 (blue) no actuation (black); (c) same as (b) but as function of the actuation period $T$ when normalised with the large-scale friction velocity that arises from the large-scale footprints for the nominal $T^{+}=100$ (red), 200 (blue). Taken from Agostini \& Leschziner (2018) with permission from Springer.

lower here, at $R e_{\tau} \simeq 850$, because the actuation causes a $30 \%$ reduction in the wall shear stress.

With the EMD performed, it is possible to quantify the direct contribution of the large scales on the skin friction. The first step is to determine the $y^{+}$-wise profiles of the large-scale shear stress ${\overline{u_{L S}}}_{L S}$, which is determined by averaging the product of large-scale velocity fields for $u$ and $v$ across the statistically homogeneous $x$ - $z$ planes and over however many temporal realisation have been stored. This distribution can then be inserted into an expression derived by Fukgata et al (2002) from a $y$-wise integration of the streamwise-momentum equation:

$$
C_{f}=\frac{6}{R e_{b u l k}}+6 \int_{0}^{1} 2\left(1-\frac{y}{h}\right)\left(-\frac{\overline{u_{L S} v_{L S}}}{U_{b}^{2}}\right) d\left(\frac{y}{h}\right)
$$

The result is that the large scales contribute $26 \%$ of the total skin friction, a contribution that is further increased by mixed-scale shear-stress fragments that correlate largescale motions with small-scale and intermediate-scale fluctuations. This level of $26 \%$ is remarkable in view of the relatively low Reynolds number, and a recent study by Agostini \& Leschziner (2019) of the unactuated channel flow at the same nominal Reynolds number has also yielded a very similar large-scale contribution.

A quantification of the indirect contribution of the large scales, by the process of modulation, is more difficult to undertake. Agostini \& Leschziner approached this challenge by constructing a probability-density function (PDF) of the large-scale fluctuations of velocity, $u_{L S}$ or skin friction, $C f_{L S}$, dividing this PDF into $5 \%$ bins, by area, and then conditionally sampling the intensity of the small-scale motions of skin friction within the bins, so as to bring out the response of the small-scale fluctuations to the intensity of the large-scale velocity fluctuations (footprints).

The manner in which the small-scale streamwise energy responds to large-scale footprints is illustrated in Fig. 13(a) by way of three PDFs for the small-scale fluctuations at $T^{+} \simeq 100$. The red and blue profiles characterise the response to the extreme $5 \%$ positive and $5 \%$ negative large-scale fluctuations, respectively, while the magenta line arises from the 5\% central PDF bin for which the large-scale fluctuations are negligible. Positive large-scale footprints cause a substantial increase in small-scale energy, while negative large-scale footprints only cause a modest decrease. This response goes hand-in-hand with a substantial thinning and a modest thickening of the viscous sublayer at positive and negative footprints, respectively. This asymmetric response, peculiar to actuated cases, is most clearly brought out in Fig. 13(b), which shows three distributions of the standard deviation of the small-scale skin-friction fluctuations as a function of the intensity of the large-scale footprints at intervals of 5\%. The black curve is for the unactuated flow, the blue for $T^{+}=200$ and the red for $T^{+}=100$. A first observation is that the actuation results in a general decline in the intensity of the smallscale fluctuations. This is expected, of course, because the actuation causes a reduction in drag and near-wall turbulence activity. A feature that is not expected is the increasing asymmetry in the modulation intensity, wherein large-scale footprints cause a higher rise in the small-scale fluctuations in positive large-scale footprints 
than the attenuation in the negative footprints, as already observed in Fig. 13(a). This asymmetry may well reflect an important mechanism by which increasingly strong outer structures impact on the drag-reduction effectiveness, as the strong amplification of the smallscale fluctuations reflect a corresponding destabilisation of the near-wall streaks and thus a tendency to increase the drag, or diminish the drag reduction. However, unfortunately, these results, while pertinent and informative in a general sense, do not allow a quantitative statement to be made on the effects of the process on the drag.

Fig.13(c) indicates a secondary mechanism by which large scales influence the drag-reduction effectiveness. Here, the red and blue curves in Fig. 13(b) are replotted as functions of the large-scale period $T_{L S}^{+}=T u_{\tau, L S}^{2} / v$. This plot therefore shows, for a fixed dimensional actuation period, the range of locally scaled periods that arise due to the strong variations in the large-scale wall shear stress provoked by footprinting. As seen, the scaled actuation period varies significantly around the nominal value. For the optimum case, $T^{+}=100$, this variability, $50<T_{L S}^{+}<115$, results in local near-wall conditions deviating from the optimal actuation conditions. Clearly, the larger the deviations, the higher the likelihood of detrimental effects on the drag-reduction effectiveness will be.

\section{CONCLUDING OBSERVATIONS}

Although the prospect of a technological realisation of spanwise wall motion in high-speed vehicles is emphatically far-fetched, the research reviewed in this paper is nevertheless more profound than just being a subject of curiosity. It is true that fascination with the turbulence mechanism that underpin the intriguingly large levels of (gross) drag reduction attained by carefully optimised sets of actuation parameters has been a major driver of the research on spanwise wall oscillations. However, one important practically-oriented outcome of the research is a list of valuable lessons on what needs to be done to the near-wall region, actively or passively, to control friction drag by reducing near-wall turbulence and thus thicken the viscous sublayer.

There are several examples of new directions of inquiry spawned by the research reviewed herein. One is an attempt to emulate the oscillatory spanwise motion passively by the use of wavy surfaces with the waves skewed relative to the flow direction (Ghebali et al, 2017). This surface topology generates a meandering motion of the near-wall flow with a component that is closely akin to a Stokes layer. Another example is the use of wallembedded disks (Ricco \& Hahn, 2013, Wise et al, 2014), either actively actuated or freely rotating, that give rise to unsteady and/or spatially meandering transverse motion. A third example is an effort to use plasma actuation to create a thin layer of unsteady spanwise motion (Duong et $a l, 2019)$, which appears capable of yielding massive drag-reduction levels (up to $70 \%$ ) for certain actuation conditions.

All the above strategies and methods share a common objective - namely, to disrupt or destabilize the processes by which turbulence is regenerated at the wall by ejections, sweeps and quasi-streamwise vortices that mix momentum close to the wall and give rise to streaks and high drag. The research on spanwise wall motion informs us that actuation is most effective if the transverse Stokes layer is confined to the viscous sublayer. The optimum mode of actuation is in the form of streamwise travelling waves at particular combinations of actuation period and streamwise wavelength $\left(T^{+} \simeq 100, \lambda_{x}^{+} \simeq 800\right)$. Unfortunately, the optimum combination of parameters is known only for low Reynolds numbers.

The dependence of the drag-reduction effectiveness on the Reynolds number is regarded as an extremely important question, and is the subject of ongoing research and debate. Most optimistically, the decline in effectiveness is slow if the effect of increasing the Reynolds number is can be described solely by an elevation of the wall-scaled log law by an invariant upward shift at all Reynolds numbers. However, some of the research described towards the end of this review makes clear that energetic large-scale motions in the outer part of the log layer have a profound influence on the turbulent structure of the near-wall layer, and this suggests a more complex set of interactions than is implied purely by a constant log-law shift.

\section{REFERENCES}

1. Agostini, L. and Leschziner, M. A., "On the influence of outer large-scale structures on near-wall turbulence in channel flow," Physics of Fluids, 26(7), 075107 (2014).

2. Agostini, L. and Leschziner, M.A., "On the validity of the quasi-steady-turbulence hypothesis in representing the effects of large scales on small scales in boundary layers," Physics of Fluids, 28(4), 045102 (2016).

3. Agostini, L. and Leschziner, M.A., "The impact of footprints of large-scale outer structures on the nearwall layer in the presence of drag-reducing spanwise wall motion," Flow Turbulence and Combustion, 100(4), pp. 1037-1061 (2018).

4. Agostini, L. and Leschziner, M.A., "The connection between the spectrum of turbulent scales and the skinfriction statistics in channel flow at $R e_{\tau}=1000$," Journal of Fluid Mechanics, 871, pp. 22-51 (2019).

5. Agostini, L., Leschziner, M.A. and Gaitonde, D., "Skewness-induced asymmetric modulation of smallscale turbulence by large-scale structures," Physics of Fluids, 28(1), 015110 (2016).

6. Agostini, L., Touber, E. and Leschziner, M. A., "Spanwise oscillatory wall motion in channel flow: drag-reduction mechanisms inferred from DNSpredicted phase-wise property variations at 
$\operatorname{Re}_{\tau}=1000, "$ Journal of Fluid Mechanics, 743, pp. 606-635 (2014).

7. Agostini, L., Touber, E. and Leschziner, M. A., "The turbulence vorticity as a window to the physics of friction-drag reduction by oscillatory wall motion," International Journal of Heat and Fluid Flow, 51, pp. 3-15 (2015).

8. Auteri, F., Baron, A., Belan, M., Campanardi, G. and Quadrio, M., "Experimental assessment of drag reduction by traveling waves in a turbulent pipe flow," Physics of Fluids, 22(11), 115103 (2010).

9. Baron, A. and Quadrio, M., "Turbulent drag reduction by spanwise wall oscillations," Applied Scientific Research, 55(4), pp. 311-326 (1995).

10. Baars, W. J., Hutchins, N. and Marusic, I., "Spectral stochastic estimation of high-Reynolds-number wallbounded turbulence for a refined inner-outer interaction model," Physical Review Fluids, 1(5), 054406 (2016).

11. Baars, W. J. and Marusic, I., "Data-driven decomposition of the streamwise turbulence kinetic energy in boundary layers. Part 1. Energy spectra," Journal of Fluid Mechanics, 882, A25-1 (2020).

12. Bechert, D. W., Bruse, M., Hage, W., Van der Hoeven, J. G. T. and Hoppe, G., "Experiments on drag-reducing surfaces and their optimization with an adjustable geometry," Journal of Fluid Mechanics, 338, pp. 59-87 (1997).

13. Bird, J., Santer, M. and Morrison, J. F., "Compliant kagome lattice structures for generating in-plane waveforms," International Journal of Solids and Structures, 141, pp. 86-101 (2018).

14. Bird, J., Santer, M. and Morrison, J. F., "Experimental control of turbulent boundary layers with in-plane travelling waves," Flow Turbulence and Combustion, 100(4), pp. 1015-1035 (2018).

15. Blesbois, O., Chernyshenko, S. I., Touber, E. and Leschziner, M. A., "Pattern prediction by linear analysis of turbulent flow with drag reduction by wall oscillation," Journal of Fluid Mechanics, 724, pp. 607-641 (2013).

16. Bradshaw, P. and Pontikos, N. S., "Measurements in the turbulent boundary-layer on an infinite swept wing," Journal of Fluid Mechanics, 159, pp. 105-130 (1985).

17. Bernardini, M. and Pirozzoli, S., "Inner/outer layer interactions in turbulent boundary layers: a refined measure for the large-scale amplitude modulation mechanism," Physics of Fluids, 23(6), 061701 (2011).

18. Choi, K.-S., "Near-wall structure of turbulent boundary layer with spanwise-wall oscillation," Physics of Fluids, 14(7), pp. 2530-2542 (2002).

19. Choi, K.-S. and Graham, M., "Drag reduction of turbulent pipe flows by circular-wall oscillation," Physics of Fluids, 10(1), pp. 7-9 (1998).

20. Dean, R. B., "Reynolds-number dependence of skin friction and other bulk flow variables in 2dimensional rectangular duct flow," Journal of Fluids Engineering - Transactions of the ASME, 100(2), pp. 215-223 (1978).

21. Du, Y. Q., Symeonidis, V. and Karniadakis, G. E., "Drag reduction in wall-bounded turbulence via a transverse travelling wave," Journal of Fluid Mechanics, 457, pp. 1-34 (2002).

22. Duong, A., Mydia, S., Corke, T. C., Hussain, F. and Thomas, F. O., "Turbulent boundary layer drag reduction using pulsed-dc plasma actuation," in Proceeding of 11th Int. Symposium on Turbulence and Shear Flow (TSFP11), Southampton (2019).

23. Fukagata, K., Iwamoto, K. and Kasagi, N., "Contribution of Reynolds stress distribution to the skin friction in wall-bounded flows," Physics of Fluids, 14(11), pp. L73-L76 (2002).

24. Gatti, D. and Quadrio, M., "Performance losses of drag-reducing spanwise forcing at moderate values of the Reynolds number," Physics of Fluids, 25(12), 125109 (2013).

25. Gatti, D. and Quadrio, M., "Reynolds-number dependence of turbulent skin-friction drag reduction induced by spanwise forcing," Journal of Fluid Mechanics, 802, pp. 553-582 (2016).

26. Gatti, D., Guttler, A., Frohnapfel, B. and Tropea, C., "Experimental assessment of spanwise-oscillating dielectric electroactive surfaces for turbulent drag reduction in an air channel flow," Experiments in Fluids, 56(5), 110 (2015).

27. Ghebali, S., Chernyshenko, S. I. and Leschziner, M. A., "Can large-scale oblique undulations on a solid wall reduce the turbulent drag?," Physics of Fluids, 29(10), 105102 (2017).

28. Howard, R. J. A. and Sandham, N. D., "Simulation and modelling of a skewed turbulent channel flow," Flow Turbulence and Combustion, 65(1), pp. 83-109 (2000).

29. Hurst, E., Yang, Q. and Chung, Y. M., "The effect of Reynolds number on turbulent drag reduction by streamwise travelling waves," Journal of Fluid Mechanics, 759, pp. 28-55 (2014).

30. Jung, W. J., Mangiavacchi, N. and Akhavan, R., "Suppression of turbulence in wall-bounded flows by high-frequency spanwise oscillations," Physics of Fluids A - Fluid Dynamics, 4(8), pp. 1605-1607 (1992).

31. Laadhari, F., "Reynolds number effect on the dissipation function in wall-bounded flows," Physics of Fluids, 19(3), 038101 (2007).

32. Laadhari, F., Skandaji, L. and Morel, R., "Turbulence reduction in a boundary-layer by a local spanwise oscillating surface," Physics of Fluids, 6(10), pp. 3218-3220 (1994).

33. Lardeau, S. and Leschziner, M. A., "The streamwise drag-reduction response of a boundary layer 
subjected to a sudden imposition of transverse oscillatory wall motion," Physics of Fluids, 25(7), 075109 (2013).

34. Lee, M. and Moser, R. D., "Direct numerical simulation of turbulent channel flow up to $R e_{\tau}=5300$," Journal of Fluid Mechanics, 774, pp. 395-415 (2015).

35. Lozano-Duran, A. and Jimenez, J., "Effect of the computational domain on direct simulations of turbulent channels up to $R e_{\tau}=4200$," Physics of Fluids, 26(1), 011702 (2014).

36. Luchini, P., "Reducing the turbulent skin friction," in Computational methods in applied sciences, J.A. Désidéri, et al., Editors, Wiley: Paris. pp. 466-470. (1996).

37. Marusic, I., Mathis, R. and Hutchins, N., "High Reynolds number effects in wall turbulence," International Journal of Heat and Fluid Flow, 31(3), pp. 418-428 (2010).

38. Mathis, R., Hutchins, N. and Marusic, I., "A predictive inner-outer model for streamwise turbulence statistics in wall-bounded flows," Journal of Fluid Mechanics, 681, pp. 537-566 (2011).

39. Moin, P., Shih, T. H., Driver, D. and Mansour, N. N., "Direct numerical simulation of a 3-dimensional turbulent boundary layer," Physics of Fluids A - Fluid Dynamics, 2(10), pp. 1846-1853 (1990).

40. Nugroho, B., Hutchins, N. and Monty, J. P., "Largescale spanwise periodicity in a turbulent boundary layer induced by highly ordered and directional surface roughness," International Journal of Heat and Fluid Flow, 41, pp. 90-102 (2013).

41. Pang, J. G. and Choi, K. S., "Turbulent drag reduction by Lorentz force oscillation," Physics of Fluids, 16(5), pp. L35-L38 (2004).

42. Pirozzoli, S. and Bernardini, M., "Probing highReynolds-number effects in numerical boundary layers," Physics of Fluids, 25(2), 021704 (2013).

43. Quadrio, M. and Ricco, P., "Critical assessment of turbulent drag reduction through spanwise wall oscillations," Journal of Fluid Mechanics, 521, pp. 251-271 (2004).

44. Quadrio, M. and Ricco, P., "The laminar generalized Stokes layer and turbulent drag reduction," Journal of Fluid Mechanics, 667, pp. 135-157 (2011).

45. Quadrio, M., Ricco, P. and Viotti, C., "Streamwisetravelling waves of spanwise wall velocity for turbulent drag reduction," Journal of Fluid Mechanics, 627, pp. 161-178 (2009).

46. Ricco, P. and Hahn, S., "Turbulent drag reduction through rotating discs," Journal of Fluid Mechanics, 722, pp. 267-290 (2013).

47. Ricco, P. and Wu, S. L., "On the effects of lateral wall oscillations on a turbulent boundary layer," Experimental Thermal and Fluid Science, 29(1), pp.
41-52 (2004).

48. Ricco, P., Ottonelli, C., Hasegawa, Y. and Quadrio, M., "Changes in turbulent dissipation in a channel flow with oscillating walls," Journal of Fluid Mechanics, 700, pp. 77-104 (2012).

49. Skote, M., "Comparison between spatial and temporal wall oscillations in turbulent boundary layer flows," Journal of Fluid Mechanics, 730, pp. 273-294 (2013).

50. Skote, M., "Scaling of the velocity profile in strongly drag reduced turbulent flows over an oscillating wall," International Journal of Heat and Fluid Flow, 50, pp. 352-358 (2014).

51. Skote, M., Mishra, M. and Wu, Y. H., "Drag Reduction of a Turbulent Boundary Layer over an Oscillating Wall and Its Variation with Reynolds Number," International Journal of Aerospace Engineering, 891037 (2015).

52. Skote, M., Mishra, M. and Wu, Y. H., "Wall oscillation induced drag reduction zone in a turbulent boundary layer," Flow Turbulence and Combustion, 102(3), pp. 641-666 (2019).

53. Touber, E. and Leschziner, M. A., "Near-wall streak modification by spanwise oscillatory wall motion and drag-reduction mechanisms," Journal of Fluid Mechanics, 693, pp. 150-200 (2012).

54. Vallikivi, M., Hultmark, M. and Smits, A. J., "Turbulent boundary layer statistics at very high Reynolds number," Journal of Fluid Mechanics, 779, pp. 371-389 (2015).

55. Viotti, C., Quadrio, M. and Luchini, P., "Streamwise oscillation of spanwise velocity at the wall of a channel for turbulent drag reduction," Physics of Fluids, 21(11), 115109 (2009).

56. Wise, D. J., Alvarenga, C. and Ricco, P., "Spinning out of control: Wall turbulence over rotating discs," Physics of Fluids, 26(12), 125107 (2014).

57. Yakeno, A., Hasegawa, Y. and Kasagi, N., "Modification of quasi-streamwise vortical structure in a drag-reduced turbulent channel flow with spanwise wall oscillation," Physics of Fluids, 26(8), 085109 (2014).

58. Yamamoto, Y. and Tsuji, Y., "Numerical evidence of logarithmic regions in channel flow at $R e_{\tau}=8000$," Physical Review Fluids, 3(1), 012602 (2018).

59. Mizuno, Y. and Jimenez, J., "Wall turbulence without walls," Journal of Fluid Mechanics, 723, pp. 429-455 (2013).

60. Zhang, C. and Chernyshenko, S. I., "Quasisteady quasihomogeneous description of the scale interactions in near-wall turbulence," Physical Review Fluids, 1(1), 014401 (2016).

(Manuscript received January 15, 2020, accepted for publication May 01, 2020.) 